\title{
Análisis de la práctica en transformación y sus efectos en los profesionales: estudio cualitativo en el programa Primer la Llar en Barcelona (Housing First) ${ }^{1}$
}

\section{Susana Batle Cladera}

Universitat de Barcelona

sbatlecl7@alumnes.ub.edu

\author{
Marta Llobet Estany \\ Universitat de Barcelona \\ mllobet@ub.edu
}

\section{Joan Uribe Vilarrodona \\ Universitat de Barcelona \\ joan.uribe@ub.edu}

\section{Jezabel Cartoixà García}

Universitat de Barcelona

jcartoga7@alumnes.ub.edu

\begin{abstract}
Artikulu honen asmoa Primer la Llar programako hiru talderen praktikaren eraldaketa-prozesua deskribatu nahi da. Housing First filosofia aintzat hartzen duen lehen programa pilotua da (2015-2018), Espainiako Estatuko Administrazio Publiko batek abiarazi duena. Bartzelonako Udala izan da ezaugarri horiek dituen programa baten antolaketa- eta teknikalidergoa bere gain hartu izandako lehenengo toki-administrazioa. Arreta-ereduaren aldaketa dokumentatzeko, metodo narratiboetan kokatutako luzetarako ikerketa erabili da. Taldeko eta banakako elkarrizketak erabiliz, narratiba-praktika jakinduriaiturri gisa landu da. Emaitzak hiru mementoren arabera aurkezten dira, eta praktikaren dimentsio indibiduala eta kolektiboa aztergai hartu. Gobernantzak eta praktikak bi norabidetan dute eragina. Artikulu honek erabaki desberdinek lagun egite profesionalaren eragin- eta eraldatze-maila ikusarazi egiten du.
\end{abstract}

\section{GAKO-HITZAK:}

Praktika-analisia, praktika profesionala, praktika-narratiba, lagun egitea, Housing First, etxegabetasuna.
El presente artículo pretende describir el proceso de transformación de la práctica de tres equipos del programa Primer la Llar. Se trata del primer programa piloto (2015-2018) con filosofía Housing First lanzado por una Administración Pública en el Estado español. El Ayuntamiento de Barcelona se convierte en la primera Administración local que asume el liderazgo organizativo y técnico de un programa con estas características. Para documentar el cambio de modelo de atención se ha utilizado una investigación longitudinal enmarcada en los métodos narrativos. A través de entrevistas grupales e individuales se ha trabajado en el relato de práctica como fuente de sabiduría. Los resultados se presentan en relación con tres momentos y exploran la dimensión individual y colectiva de la práctica. La gobernanza y la práctica están influenciadas bidireccionalmente. Este artículo visibiliza los niveles a través de los cuales las diferentes decisiones afectan al acompañamiento profesional y guían su transformación.

\section{PALABRAS Clave:}

Análisis de la práctica, práctica profesional, relato de práctica, acompañamiento, Housing First, sinhogarismo.

\footnotetext{
${ }^{1}$ Esta investigación ha sido encargada y financiada por el Ayuntamiento de Barcelona. Agradecemos a los profesionales de los equipos, así como a los coordinadores y responsables organizativos, la posibilidad de realizar esta investigación.
} 


\section{Contextualización de la investigación}

La práctica profesional es un tema de candente actualidad. Ante cuestiones como ¿qué hacen los profesionales del ámbito social?, ¿de qué manera se responde a determinadas situaciones?, ¿por qué se toman determinadas opciones y no otras?, a menudo se responde con descripciones retóricas y poco concretas, que no permiten evaluar los efectos que producen las prácticas. Esta respuesta nos señala la falta de reflexión y autocrítica respecto a nuestra acción profesional. No existe una definición unificada, aunque tampoco debería existir como tal, pues la singularidad es una de las claves para poder adaptarse a las personas que acuden al servicio o al encuentro del profesional. En cualquier caso, es inquietante reconocer que nuestra mirada se centra más en los sujetos atendidos que en nuestro quehacer.

En el siguiente artículo se presentan los resultados de un estudio longitudinal que ha durado tres años, centrado en el análisis de la práctica profesional de los equipos del programa piloto Primer la Llar (20152018). Es un programa lanzado por el Ayuntamiento de Barcelona y basado en la filosofía Housing First. La administración realiza el encargo de una evaluación cuantitativa y otra cualitativa. La cualitativa ahonda en dos elementos de completa actualidad. Los efectos que produce el modelo Housing First en los y las participantes y el análisis de la práctica. Este artículo se centra en este segundo análisis, con el objetivo de conocer el proceso de transformación de la práctica entre dos modelos de atención.

Tabla 1. Investigaciones asociadas al programa piloto Primer la Llar

\begin{tabular}{|c|c|c|}
\hline \multicolumn{3}{|l|}{ Cualitativa } \\
\hline Cuantitativa & $\begin{array}{l}\text { Los efectos del } \\
\text { programa sobre el } \\
\text { proceso de recuperación } \\
\text { de los participantes. }\end{array}$ & $\begin{array}{l}\text { Análisis de la práctica } \\
\text { profesional en proceso } \\
\text { de transformación } \\
\text { entre dos modelos de } \\
\text { atención. }\end{array}$ \\
\hline
\end{tabular}

Fuente: Elaboración propia.

Los profesionales de Primer la Llar deben atender desde el modelo Housing First pero no disponen de pautas claras, de referentes cercanos o bibliografía específica. Se encuentran a caballo entre dos modelos. Del anterior modelo escalonado - “continuum of care" o Treatment First- tienen la experiencia de lo que hicieron, pero la literatura publicada o las investigaciones sobre su funcionamiento es prácticamente inexistente (Padgett, 2012). Tras un análisis más bien empírico se entiende que la metodología de trabajo es conductual y su filosofía parte de una premisa meritocrática (Sahlin, 2005). El auge de la atención puesta en el modelo Housing First ha brindado la oportunidad de investigar en el ámbito del sinhogarismo, con el fin de poder ofrecer una atención cada vez más digna y profesionalizada.

Las investigaciones centradas en el fenómeno del sinhogarismo están, en su mayoría, basadas en programas Housing First. Su llegada a Europa, la creación de la Feantsa (1989) y algunas investigaciones pioneras en nuestro país (Cabrera, 1998; 2000), pusieron en entredicho el silencio reinante. En palabras de Llobet y Aguilar: “La literatura escrita sobre el modelo es extensa, pero hay muy poca información sobre la intervención y la práctica asociada al modelo" (2016: 26).

Aportamos aquí un ingrediente novedoso, pues la práctica profesional se empieza a analizar recientemente en el ámbito social. Existen antecedentes en el campo de la educación, donde cuenta con varias décadas de trayectoria y ha demostrado sus beneficios en la progresión de la acción, en la investigación de los procesos y en la profesionalización (Barbier, 2004). Las prácticas como unidad de análisis en el sinhogarismo aparecen en el último lustro (Andvig, Sælør y Ogundipe, 2018; Barrenger, Stanhope y Atterbury, 2017; Buxant, 2018; Devine y Bergin, 2020; Fenwick et al., 2019; HomelessLink, 2017; Lo Sardo, 2016; Rosa, 2019; Sacco, 2020; Samhsa, 2010; Stanhope, 2012; Vánĕ, 2020; Anderson-Baron y Collins, 2018; Hasford et al., 2019; Watson et al., 2018) evidenciando su papel principal en la transformación de los servicios de atención.

Esta investigación, basada en métodos narrativos, mencionará al modelo anterior a pesar de estar únicamente centrada en el entrante. Esto se debe a que los profesionales utilizan la comparación entre ambos para diferenciar lo que hacían de lo que hacen, ratificando los cambios producidos en sus formas de hacer, pensar e identificarse (Lafortune, 2009). Analizar la práctica es sinónimo de querer mejorar y dejarse atravesar, como veremos más adelante, tanto en el ámbito personal como profesional (Alföldi, 2017).

\section{Marco teórico}

\section{1 ¿Qué es la práctica?}

La práctica puede definirse como el ejercicio voluntario de una acción que modifica el entorno al poner en diálogo competencias y experiencias (Alföldi, 2017). Barbier (2011) aborda la cuestión desde su verbalización y afirma que la práctica profesional es aquello que los profesionales dicen que hacen. Relatar la acción es muy útil porque clarifica lo que hacemos, ayuda a descartar lo que no hacemos y también a consensuar con el resto de las personas implicadas lo que queremos hacer. Es un despliegue de saberes técnicos, académicos y/o experienciales en un contexto concreto.

La acción de los profesionales en el ámbito social está siempre en relación con la persona a la que se informa, atiende, acompaña o, en el peor de los casos, sobre la que se interviene. Es un hacer en relación que implica intersubjetividad (Barbier, 2011). Esta necesidad de pensar la práctica respecto a un 
vínculo influirá, como veremos posteriormente, en la relación establecida entre las partes.

Para Barbier (1999), la práctica puede ser entendida como una intención puesta en práctica, como un algoritmo de operaciones para conseguir un resultado, como una habituación o como una transformación de una realidad a otra. Desde aquí encontramos diferentes disciplinas desde las que se ha abordado la práctica como la educación con el razonamiento inconsciente (Perrenoud, 2012), la psicología con los esquemas de acción o estructuras cognitivas (Piaget, 2000), la sociología desde el habitus o estructuras estructurantes (Bourdieu, 2007; San Martín y Quilaqueo, 2012), la práctica situada (García-Molina y Saéz, 2011; Garneau y Namian, 2017; Gherardi, 2008; Goffman, 1991); un producto elaborado por subjetividades en interacción (Blumer, 1982; Pessin, 2004; Stanhope, 2012) o como un elemento artesanal moldeado lentamente a través del paso del tiempo y la autocrítica (Navarro, 2017; Sennett, 2009). También desde la antropología como estrategia ante la improvisación en interacción (Laborde, 1999), o como corporal al encarnarse en un agente (Esteban, 2004).

En nuestro caso, tomamos la noción de práctica como la actividad de un profesional que busca hacer efectiva la transformación hacia el nuevo modelo de atención. La filosofía Housing First indica ocho principios y la experiencia recogida en la literatura nos habla, grosso modo, sobre fidelidad, éxitos y el derecho a la vivienda. Ahora bien, todo esto ¿cómo se consigue? ¿Qué fórmula mágica hay que accionar para que las personas disfruten del autogobierno de su vida?

El trabajo homogéneo, con maneras de hacer estancas, prefijadas estaría ligado a un trabajo por habituación (Barbier, 1999). Por lo tanto, para trabajar por la transformación hay que empezar por reconocer la incomodidad, "ese espacio fronterizo, inestable, sujeto a negociaciones y estructurado por los modos en que se construye hegemonía y valor" (Danel, 2020: 8). Ceder a la incertidumbre y esperar, desde un amplio abanico de posibilidades, a que surjan nuevas realidades, comunicaciones, relaciones (De Robertis, 2013). Visibilizar las cuotas de poder que ostenta un profesional por el simple hecho de serlo y cómo estas aumentan a medida que aumenta la vulnerabilidad del otro, es otro ejercicio de transformación de la práctica. Ser consciente de esta jerarquía social da la oportunidad de reflexionar acerca de ello, de repensarse en relación y transformar ese espacio/tiempo de privilegio. Tiempo y espacio, dos dimensiones que marcan una diferencia esencial entre ambos modelos de atención (Batle et al., 2020).

Con la profesionalización y la consolidación del saber-experto se ha expropiado "a la población la posibilidad de definir su propia problemática y ser ella misma la protagonista de sus propias soluciones" (Ávila et al., 2019: 134). Para devolverles esta capacidad y considerarlas expertas en sí mismas es necesario hablar de la práctica dialógica o praxis en sentido Freiriano (2005). Si los individuos pueden nombrar su mundo tienen la posibilidad de transformarlo. Para ello se necesita confianza, horizontalidad, esperanza, transparencia, humanización, una relación ética alejada de la conquista y altas dosis de paciencia e incomodidad (Freire, 2005; Meirieu, 1998).

\subsection{Análisis de práctica}

Analizar la práctica sirve para comprender el porqué y el cómo. Es hacerse responsable del acto profesional a través de la reflexión conjunta y del repaso de las acciones pasadas (Guigue, 2002; Vergnaud, 1996). En este proceso, los equipos de trabajo realizan una toma de conciencia de hábitos, motivaciones, creencias y usos del lenguaje y del cuerpo. El profesional se

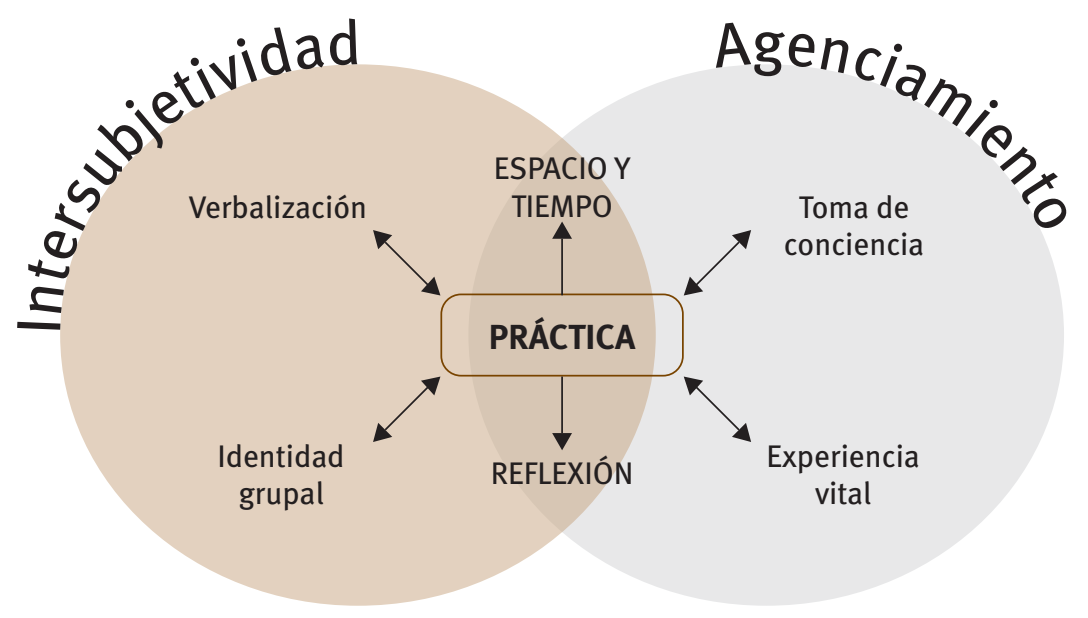


contextualiza en una historia y busca, de alguna manera, salir de ahí, presentarse en su realidad de forma diferente. Se cuestiona sobre el origen y los límites. Cuestiones que a menudo permanecen ocultas en el inconsciente. Visibilizarlas, y especialmente compartirlas, activa procesos de agenciamiento.

La necesidad de iniciar este análisis puede ser propia del equipo, del proyecto o una demanda de la entidad. En este último caso suele tratarse de una evaluación de la práctica, algo con lo que conviene no confundirse (Barbier, 1993; 1999).

Un dispositivo de análisis de práctica es un espacio de trabajo colectivo en el que se reúnen diversos profesionales y comparten sus experiencias. Realizan un trabajo reflexivo tanto individual como grupal que permite identificar y tomar consciencia de cómo perciben e interpretan situaciones además de ir entretejiendo el rol que desempeñan. Son procesos organizados en base a una metodología concreta -observación, escenificación, discursividad o escritura-. Emerge un trabajo colaborativo a través de las preguntas, hipótesis y recuerdos que deviene formativo, a pesar de no ser su intención principal. Estos intercambios generan saber-hacer, acumulando experiencias sobre las que basar nuevas acciones, una especie de mémoire professionnel (Marcel et al., 2002).

Entre los intereses del dispositivo podemos destacar la identificación de tendencias ideológicas. Los sistemas de valores que operan en los equipos de atención suelen darse por sentados. Sostienen una distribución de poderes concreta basada en el hábito del "siempre se ha hecho así". A través de la reflexividad sobre y durante la práctica pueden visibilizarse y cuestionarse estas derivas. Descentralizar ciertas verdades abre nuevas posibilidades epistemológicas que cuesta sostener. La transformación de la práctica requiere una exposición emocional importante que puede llegar a sobrepasar a los profesionales. Algunos estudios realizados con personas que sufren una fuerte vulnerabilidad muestran cómo los profesionales tienen más posibilidad de sufrir trauma secundario o burnout (Rogers, George y Roberts, 2020). Si a esto le sumamos la asunción del uso del poder ejercida con antelación aparece la necesidad de acompañar a los profesionales a través de formación y espacios de cuidados, tanto formales como informales (Cosentino et al., 2020; Lenzi et al., 2020).

Reflexionar sobre las acciones pasadas y reflexionar en el momento en que se realizan (Schön, 1998) implica reconocer la experiencia. La práctica reflexionada se presupone a personas críticas y autónomas que se guían a través de sus principios y valores (Eisner, 1998). Esta experiencia en entornos formas de adquisición de saber y competencias, entiende a cada individuo como un universo de experiencia y pone en valor a la persona como generadora de recursos propios para su aplicación (Delory-Momberger, 2014). La experiencia conjugada con la práctica profesional configura la identidad del reconocerse y ser reconocidos (Ricoeur, 1990).

Detrás de la atención social siempre hay un equipo. Es por eso por lo que hablamos de compartir y reflexionar conjuntamente. Esta reflexión sobre la acción es formativa y produce identidad (Barbier, 1999), fortaleciendo las habilidades específicas requeridas, aumentando el nivel de especialización, facilitando aclaraciones sobre límites y desafíos y finalmente desarrollando capacidades para comprender y adaptarse a las demás - tanto personas atendidas como otros profesionales- (Fablet, 2004).

Elegir el camino de la práctica reflexionada no es fácil, requiere un compromiso personal y laboral honesto, flexible y creativo. Muchas veces artesanal (Navarro, 2015). Requiere deseo, sed de aprendizaje y trabajo. Hay que prepararse para asumir $y$ reconocer errores, sentirse cuestionado y mantener las ganas de seguir mejorando (Alföldi, 2017).

\subsection{Relato de práctica}

El relato de práctica se enmarca en la fenomenología, forma parte del método biográfico o narrativo y está enraizado en la conversación, la documentación y la observación participante. Busca ahondar en la experiencia a través del repaso de hechos pasados y las vivencias experimentadas por los profesionales (Bertaux, 1976). Si el relato de vida se centra en la trayectoria vital de una persona y en documentos producidos por ella, el relato de práctica es un método para conocer lógicas concretas. Tiene la intención de mejorar las habilidades de un equipo y combatir las tendencias al etnocentrismo o corporativismo (Grimaud, 2003).

La capacidad de un equipo para identificar, reconocer y repasar los eventos pasados, las dinámicas y las decisiones, es el principal facilitador de la técnica para promover la transformación y hacer emerger los significados que los profesionales dan a acciones, situaciones, relaciones y acontecimientos. La función narrativa de este método está íntimamente ligada con la producción identitaria del grupo (Ricoeur, 2004). Es por eso por lo que iniciar este trabajo de transformación, compartiendo información, sincerándose y mostrando también partes íntimas de cada miembro ayuda a verse reflejado en el otro y consolida a los equipos en el viaje de la indagación y experimentación de nuevas maneras de hacer. Así pues, la búsqueda del equilibrio entre lo que el profesional sabe y lo que se hace conjuntamente sirve para que puedan "justificar sus pretensiones y respaldar sus juicios” (Santibáñez, 2012: 32).

\section{Metodología}

Esta investigación cualitativa y longitudinal ha durado tres años y se enmarca en los métodos narrativos, pues pretende generar conocimiento a 
partir de experiencias vividas por las personas en relación con su contexto sociohistórico, político, económico y cultural. Sacar a la luz los aprendizajes hechos obliga a observar y documentar los elementos que han ido dialogando con la práctica y su evolución. Se centra en el proceso, por eso las herramientas elegidas permiten flexibilidad para captar el movimiento propio de la transformación.

A diferencia de la evaluación de las prácticas, analizarlas es realizar un ejercicio profundamente cualitativo que busca comprender. La investigadora, que pone el cuerpo durante el proceso, se entrega a la escucha del otro con empatía, sin juicio. Crea un clima en el que se respira un profundo respeto entre las participantes, dando lugar a reflexiones que nacen de las entrañas, muestras de sinceridad que ponen al descubierto partes muy íntimas, tanto individuales como grupales. Desde ese lugar y a través del relato, se reconstruyen las derivas de la práctica durante estos tres años.

El análisis de práctica puede desarrollarse desde diversos paradigmas (Marcel et al., 2002). En este caso se ha elegido el socioconstructivista, uno de los más aceptados y utilizados para analizar las prácticas docentes. Según Lafortune (2009), este enfoque es oportuno para acompañar en la adopción y escenificación de un nuevo esquema de actuación. El vacío bibliográfico al que hicieron frente los equipos hizo imprescindible el uso de algunos métodos y herramientas de la teoría fundamentada. Concretamente, estos equipos han tenido que ir construyendo a medida que se han encontrado frente a situaciones nuevas. Han puesto en práctica la reflexión y la creatividad, la experiencia adquirida en situaciones pasadas y una visión a futuro de hacia dónde caminar. Al igual que el bertsolari improvisa y al mismo tiempo construye la obra, los equipos, al accionar ante situaciones inéditas, van construyendo una nueva práctica (Bardón, 1998). Han improvisado ante la urgencia como lo haría un músico de jazz en una jam session (Haidet, 2007). La teoría fundamentada, al no partir de hipótesis que validar, busca comprender e interpretar el dinamismo y adaptabilidad de la práctica entre aquello que era y lo que quiere llegar a ser.

El papel de las investigadoras ha sido clave en el proceso, pues han acompañado a esa producción de información. El formato de entrevista relato va más allá de la pregunta respuesta, invita a una conexión con el otro y aporta a la identidad grupal. Los espacios destinados a las entrevistas grupales han sido a la vez espacios de reflexión y autoformación donde han emergido otros saberes más allá de los presupuestos formativos o laborales. Tal como dice Fablet (2007: 41): "se invita a los sujetos a involucrarse en el análisis, es decir a trabajar en la co-construcción del significado de sus prácticas y/o la mejora de las técnicas profesionales".

Se ha apostado por el relato de práctica como estrategia innovadora en el ámbito de las ciencias sociales por la cantidad de beneficios que aporta. La verbalización de la experiencia pone atención en la creatividad, la intuición y la paciencia (Desgagné, 2005). Volver a conectar con limitaciones y su resolución sirve como ejercicio autoformativo (Bertaux, 2005; Desgagné, 2005) y como recopilación del repertorio de acciones (Schön, 1998; Schön y Argyris, 1995; Schön y Rein, 1994).

La muestra está compuesta por tres equipos de atención; dos de atención social y uno de atención psiquiátrica. Además, han participado los técnicos del ayuntamiento responsables del programa. Debido al carácter longitudinal y a los cambios en la composición de los equipos, la muestra ha ido variando. En total, han participado cuarenta personas. De uno de los equipos de atención social participaron un total de doce profesionales, del otro fueron nueve. Del equipo de atención psiquiátrica colaboraron once, del ayuntamiento, cuatro y de otros servicios externos, tres profesionales más. Los equipos multidisciplinares estaban compuestos por las categorías profesionales que muestra la Tabla 2.

El trabajo de campo se realizó en tres fases. La primera se desarrolló entre marzo y diciembre del 2017 a través de entrevistas tanto grupales - abiertas y adaptadas al relato de práctica-como individuales -semiestructuradas-. Las entrevistas grupales se realizaron una vez al mes durante tres meses a los equipos sociales. El equipo de atención psiquiátrica realizó una entrevista grupal. En cada entrevista se han tratado temas diferentes con relación a la construcción de una nueva manera de hacer.

La segunda duró algunos meses más debido a una reestructuración del calendario general de la investigación y se llevó a cabo entre junio del 2018 y febrero del 2019. Se realizaron un total de catorce entrevistas, trece grupales y una individual. De las grupales, se hicieron doce a los equipos sociales y una al equipo de atención psiquiátrica. La temática se centró en la práctica orientada a la recuperación y la reducción de daños.

Tabla 2. Características de la muestra

\begin{tabular}{c|c|c|c}
\hline Equipo de atención social 1 & Equipo de atención social 2 & Equipo de atención psiquiátrica & Ayuntamiento \\
\hline 2 Educadoras social & 1 Educadora social & & \\
1 Trabajadora social & 5 Trabajadoras sociales & 3 Psiquiatras & 2 Psicólogos \\
2 Integradoras sociales & 3 Integradores sociales & 2 Enfermeras & 1 Jefe de servicio \\
1 Par igual (peer worker de baja) & 1 Par igual (peer worker) & 2 Coordinadoras & \\
1 Coordinadora de equipo & 1 Coordinadora de equipo & & \\
2 Responsables de proyecto & 1 Responsable de proyecto & & \\
\hline
\end{tabular}

Fuente: Elaboración propia. 
Finalmente, la tercera fase se realizó entre los meses de diciembre del 2019 y abril del 2020. En total se realizaron ocho entrevistas; seis fueron individuales y dos por parejas. En ellas se abordó la valoración general sobre la experiencia piloto en lo relativo a la práctica profesional y a la gobernanza.

Toda la información fue registrada en audio con consentimiento previo y guardando el anonimato. A continuación, se transcribieron de forma fidedigna añadiendo todas las anotaciones recogidas en el cuaderno de notas con relación a las observaciones y conversaciones surgidas al apagar la grabadora.

Los datos se trataron a través del programa de gestión de datos cualitativos Atlas.ti 7 y 8 . El libro de códigos se diseñó a partir de elementos deductivos utilizados para el diseño de los guiones e inductivos, que finalmente fueron los más útiles.

Tras cada entrada a campo se ha hecho una devolución a los equipos en relación con los resultados del relato de práctica. Tanto en la primera como en la última fase se realizó una devolución formal a todos los informantes del programa, seguida de un debate. Para la fase intermedia se realizó una devolución al inicio de cada sesión, que dio lugar a una reflexión de segundo orden en cada uno de los equipos sociales.

Por otro lado, la investigación ha tenido otra fase de trabajo de campo realizada entre 2017 y 2018 dedicada exclusivamente a la recogida de datos internacionales. Se visitó a los equipos de Diogène en Montreal (Canadá), Chez Soi-d'abord en Marsella (Francia) y Casas Primeiro en Lisboa (Portugal). La intención fue poder recoger la experiencia de estos programas en su fase de piloto para entender las barreras, dificultades, facilitadores y estrategias con las que convivieron los equipos. En total se realizaron nueve entrevistas semiestructuradas individuales y una grupal. A excepción de las devoluciones, el tratamiento de los datos ha seguido el mismo proceso que los recogidos en el programa Primer la Llar.

\section{Resultados}

Se presentan los resultados siguiendo la estructura del trabajo de campo en tres momentos clave en la transformación de la práctica. De esta forma se pueden seguir los cambios de forma temporal. En primer lugar, se aborda cómo los equipos saben más de la atención anterior que del modelo Primer la Llar. En segundo lugar, se analiza la transformación, cómo los profesionales son capaces de echar la vista hacia atrás y ver los recorridos. Finalmente, cómo pueden mirar desde la distancia y valorar el trabajo hecho a través de una crítica fundamentada. Estos tres momentos están atravesados por las dos dimensiones que Barbier (2011) le reconoce a la práctica, una individual referente a su asimilación e interpretación en tanto que experiencia singular y otra colectiva por su uso dialógico, práctico e interactivo.
En la dimensión individual están las personas que componen el equipo en calidad de persona y en calidad de profesional. El proceso de cambio de modelo tiene implicaciones en ambos estatus.

\subsection{Fase inicial: herencia del modelo anterior}

\subsubsection{Dimensión individual del cambio de práctica}

\section{Personal}

Durante la primera fase de entrevistas, los informantes fueron reconociendo cómo algunos elementos relativos a su intimidad habían sido de utilidad al acompañar a las personas. Esto no quiere decir que desde el modelo tradicional los profesionales consigan eliminar parte de su personalidad y presentarse asépticos en el trabajo, pero la normativa y los protocolos de actuación sí que invitan a trabajar de una forma más homogénea y pautada, eliminando parte de la creatividad que han tenido que invocar estos equipos. Uno de los profesionales explica que tiene una patología visual a partir de un accidente de tráfico en la infancia. Las dificultades vitales a las que se ha ido enfrentado durante los años le han ido generando rabia, frustración, ha maldecido ciertas situaciones y finalmente ha decidido "tirar p'alante". Este proceso le ha permitido empatizar con las personas que tienen algún tipo de diversidad funcional. También han aparecido los procesos migratorios, la precariedad económica o personas cercanas con malestares mentales. Un profesional utiliza una de las frases que le decía su madre cuando los alimentos escaseaban, compartiéndola así con las personas. Extraer elementos propios de la experiencia vital individual no queda en esa frase o en esa empatía, sino que se comparte entera, contextualizada. Es un regalo que se entrega a las participantes para que puedan hacer el uso que deseen. Lo que sí sabemos con certeza es que se trata de un hilo que, al igual que el de Ariadna, sostendrá la confianza "pase lo que pase".

Compartir estas vivencias ha iniciado un trabajo de proximidad. Ciertamente esta era una de las características de la práctica desprendidas de los principios. Pero utilizar la empatía, poniéndole nombre y compartiéndola con los participantes, ha ayudado a hacer efectivo este trabajo de proximidad. Fue uno de los primeros pasos para conseguirla según los componentes enunciados por Pascale Jamoulle (2007).

En el programa Primer la Llar, al encontrarse, ambas partes necesitan ser reconocidas por la otra. Este reconocimiento mutuo crea el camino hacia una horizontalidad buscada. Para ello cada parte ha tenido que ceder en el plano de las reticencias. Los profesionales encontraron en sus procesos terapéuticos individuales o formaciones en gestión emocional ciertas claves para empatizar e ir desvelando la inutilidad de la verticalidad. De 
hecho, un miembro del equipo explica cómo la capacidad para discernir qué es mío y qué es tuyo en la emocionalidad que emerge del encuentro viene a sustituir un establecimiento de límites férreo entre el tú y el yo.

Por otro lado, a medida que los informantes han ido reconociendo prácticas mecánicas en su trayectoria laboral, han podido verbalizar, en grupo y haciendo equipo, la incomodidad silenciada y sostenida en el tiempo. Verbalizarla ha sido terapéutico para ellos; así lo afirman al haber descubierto otra verdad sobre la acción social -entiéndase aquí verdad como otro punto de vista posible para nombrar la realidad-.

\section{Profesional}

El mandato recibido por los equipos desde la Administración fue claro: libertad para ensayar, probar cosas nuevas, siempre que fuese en beneficio de la persona. Esta libertad, junto con la falta de marco sobre cómo actuar, fue lo que conectó la dimensión individual con la colectiva. Permitió a los equipos trabajar con coherencia respecto a sus valores personales. Posteriormente se trabajó en largas reuniones de equipo, dando lugar a los valores grupales. Un proceso tremendamente bonito en el que el corazón y la razón pudieron dialogar pausadamente, sin presiones.

Los profesionales pasaron de un primer momento en el que se posicionaron fuertemente en contra de la manera de hacer anterior, para llegar a entender que esa práctica eran los cimientos que les permitirían construir otra manera de relacionarse. De nada sirve denostar el modelo anterior y renegar de la herencia pues se cae entonces en esa circularidad propia del ámbito social que nos mantiene anegados en una falta de herencia cultural propia. No se trata de modelos, se trata de metodologías y para transformarlas se hace necesario trabajar en base a una práctica anterior y reflexionarla, como se verá en la dimensión colectiva.

Los profesionales expresaron consternación al descubrirse juzgando a los participantes. Por ejemplo, una trabajadora social explica su reacción al ver el primer carro de la compra de un señor con cervezas: “¿Tenemos que pagarle la Xibeca?”. También al verse recurriendo a dinámicas de atención que les facilitaban el trabajo pero que no eran la mejor opción para los participantes: “¿Esto es bueno para mí o para la persona?".

Es un ejercicio de ir reconociendo los efectos que tienen sobre uno mismo las decisiones que se toman o los pensamientos mecánicos que surgen. El trabajo de proximidad activa cierta emocionalidad que el trabajo pautado contenía. Las conversaciones sinceras, los paseos por la ciudad sin prisas, una intención a la espera de ser expresada por los participantes, todo eso activa una implicación emocional diferente. Tener ganas de llorar, reír, celebrar, enfadarse, pero todavía no atreverse a decirlo porque "no es correcto" ¿Está bien sentir cosas? Discernir si esto es bueno o malo $y$, finalmente, entender que las dicotomías tampoco son la respuesta, invita a que el rol profesional viaje por un camino de descubrimiento íntimo.

El cambio de rol necesita tiempo, espacio, contradicciones, dificultades, miedos, riesgos, etc. Es un cambio de etapa, como lo es la adolescencia ¿Podría un adulto en ciernes trascender a la edad adulta sin todas esas divagaciones acerca de su identidad?

\subsubsection{Dimensión colectiva del cambio de práctica}

El equipo no es la suma de las partes, sino que requiere de una construcción conjunta. Estamos aludiendo a cierta consciencia grupal capaz de impulsar un proceso de transformación conjunto. El coordinador aparece como eje desde el que pivota el trabajo y consolida el todo.

Ante la falta de marco, asumir este papel no ha sido fácil y los coordinadores de los equipos también han tenido que explorar cómo acompañar a los profesionales a transitar esa crisis de identificación escenificada en dificultades para encontrar consenso. Uno de los equipos tuvo más dificultades que el otro, y por ello ganó también más aprendizajes. Pero lo que ambos establecieron como requisito fue el acompañamiento al equipo a través de sesiones de supervisión mensual. Esto palió el sentimiento de soledad que emergió en esta primera fase al no tener con quien hablar, a quien preguntar o en quien reflejarse.

Para sentirse más seguros en el acompañamiento y mostrar coherencia entre profesionales, establecieron reuniones pre y post visita, unificando la línea de acción y valorando posteriormente cómo habían implementado aquello que previamente habían decidido. Además, estos espacios también se utilizaron para verbalizar las estrategias que habían utilizado al responder a situaciones que no habían previsto. Estas conversaciones se mantuvieron en espacios informales como bares, caminando por la ciudad o en el transporte público. Pero luego todo era compartido con el equipo en reuniones formales. Era tanta la necesidad de expresarse, de buscar complicidad y de sentirse seguros en lo que se hacía que al principio las reuniones fueron muy largas y apasionadas. Los coordinadores tuvieron que activar diferentes estrategias. Uno de los equipos se vio privado de los espacios formales porque la tensión fue en aumento; esto les llevó a explorar opciones informales que los suplieran. El otro equipo tuvo que acotar diferentes reuniones para distintos temas, pudiendo recoger así las diferentes preocupaciones y necesidades. Ambos se dieron cuenta de la enorme importancia de compartir las experiencias y los sentimientos. Ante situaciones no previstas y en pos de conservar esa coherencia buscada, los 
profesionales utilizaron la técnica de posponer las respuestas cuando verdaderamente no las tenían.

Tratar estos temas en el equipo y debatirlos hasta encontrar una respuesta pensada desde la óptica de las personas es el inicio de la construcción de un lenguaje, dinámicas y estrategias comunes. Despliega transparencia y entreteje confianza. Evidencia que la responsabilidad ante las decisiones que se han tomado entre todos también es de todos. Sienta las bases del dispositivo.

Otro de los temas que preocupó a los profesionales durante esta primera fase fue la mirada pública puesta sobre el programa. De hecho, la incertidumbre propia del acompañamiento sin pautas claras de actuación pesó sobre los equipos que querían cumplir con expectativas tanto ajenas como propias.

En relación con la coordinación del equipo de salud, destaca cierta dificultad por encontrar un encaje de miradas. La herencia cultural de atención de ambos campos todavía diverge en algunos puntos. La falta de formación específica de los equipos, tanto por separado como conjuntamente, incrementó esa diferencia. En esta primera fase los equipos sociales atendían mucho desde la urgencia. Todavía sentían mucha responsabilidad por los actos ajenos y dificultad para dejar a los participantes fluir en la cotidianeidad de sus vidas. Reclamaban al equipo de salud trabajar en esa misma clave de urgencia que ellos sentían como necesaria.

\subsection{Mitad del programa: activación del cambio de práctica}

\subsubsection{Dimensión individual del cambio de práctica}

\section{Personal}

Tal como se ha mencionado, es un trabajo de proximidad, pues el equipo abandona la institución, el escritorio, y se acerca no solo a las casas de los participantes, sino también a su comunidad y cotidianeidad más íntima. Realizan un acompañamiento no tanto funcional sino existencial -aparece aquí la espiritualidad-. Es un trabajo que invita a relacionarse desde otros roles y despierta en ambas partes sentimientos de miedo e indefensión. Los profesionales viven una exposición propia del etnógrafo (Jamoulle, 2007; Baczkowska, 2017), pues dejan de considerar a las personas como beneficiarias pasivas de un servicio para entenderlas como informadoras-colaboradoras.

Los equipos han verbalizado sentirse frágiles al presentarse "desnudos". Han reconocido haberse equivocado; también han reconocido no saber qué hacer, han preguntado a las personas qué harían ellas, reconociendo que el otro también es experto y que el saber de la acción social también se construye en interacción. De hecho, en las visitas se conversa y se comparten aficiones, gustos musicales, culinarios, viajes, detalles vitales con significancia en el presente, rincones de la ciudad mágicos, etc., sin necesidad de guiones, preguntas o inquisiciones. No se trata de un trabajo poco pautado o sin objetivos socioeducativos, se trata de un trabajo basado en la vida cotidiana. Su incontrolabilidad hace imprescindible esperar a que de ella emerjan elementos que den "pie a". Se trata de una acción social inductiva. Con todo eso, la proximidad aumenta y también la coherencia. Los trabajadores entienden que poner parte de su bagaje experiencial personal al servicio de la relación es un valor añadido a su rol profesional.

Los profesionales se han dado cuenta de que no trabajan en relación con éxitos o fracasos sino que trabajan en procesos. La evidencia de si el acompañamiento da frutos en la recuperación de la persona solo puede verse a largo plazo. A corto, les quedan las impresiones y reacciones, elementos que han demostrado ser útiles en este programa. Los equipos apuntan que si algo es útil para la persona, es válido. Por lo tanto, esta exposición a través de la que se consolidan la confianza y la horizontalidad es válida y entra a formar parte de la metodología de trabajo. Podríamos hablar de una metodología del afecto que revierte otras propuestas más asépticas de encuadre de la relación.

\section{Profesional}

¿Qué sucede cuando reconozco toda esta parte personal mientras trabajo? Directamente, que mi rol profesional queda interpelado y, de alguna manera, está obligado a hacer frente a críticas y autocríticas. Destaca la pregunta que se hacen al terminar las visitas: “¿habré hecho bien?”; “¿ha servido de algo?”. En esta segunda fase sucede algo diferente a la primera, dejan de juzgarse en términos maniqueos y despliegan la conversación como estrategia de acción. Preguntan, escuchan, se expresan e informan. Les preguntan a los participantes las dudas que les surgen: “¿cómo fue la visita del otro día?”; “¿estuviste cómodo conmigo?”; “yo me sentí...”. Si la persona es la que decide y está en el centro del acompañamiento, es inevitable que se convenga la forma en la que se acciona la práctica profesional conjuntamente.

Una de las estrategias que destacan en esta fase, y que muestran la permeabilidad entre los estatus personal y profesional, es la de ponerse ellos mismos como ejemplo. Durante una de las visitas, un participante le pregunta al profesional si a él también le preocupa no llegar a final de mes, si también tiene que calcular en qué gasta el dinero. El profesional le explica situaciones vividas. Hacer visible la parte cotidiana de un profesional, sin tapujos, es un acto de coraje y de incalculable valor para la recuperación de las personas.

Que un profesional y un participante sean de la misma etnia, compartan algún estigma o hablen 
un mismo idioma hace que la identificación sea más rápida. Pero esta cercanía también entraña dificultades. En esta segunda fase aparecen problemáticas estructurales como el machismo o el racismo. Las trabajadoras mujeres han relatado situaciones tensas al acompañar a hombres. Por otro lado, compartir alguna característica en términos más dolorosos - abusos, violencias, depresionespuede llegar a despertar procesos emocionales que se creían cerrados. La pregunta es si en el modelo anterior no sucedía. Evidentemente, todos somos afectables por la realidad que nos rodea, pero los límites en la relación, un hacer más pautado y la falta de espacios grupales de expresión de sentimientos los invisibiliza.

El beneficio de esta relación de confianza fue observable en la satisfacción laboral de los equipos que dedicaron tiempo a cambiar sus esquemas mentales previos, se lanzaron a utilizar la creatividad y aunaron esfuerzos ante situaciones complicadas. Otro de los beneficios que destacan en esta etapa es cómo los participantes acaban ayudando también a los profesionales. En este programa los profesionales han tenido que ejercer el músculo de recibir, que profesionalmente estaba un tanto atrofiado. Se han dejado invitar a cafés, a comidas - no sin ciertos dilemas-. Uno de los profesionales relató cómo un participante le estuvo asesorando durante un tiempo sobre los teléfonos móviles que había en el mercado, mejores opciones, precios y prestaciones. Le costaba decidirse a comprarse uno a pesar de que el suyo era muy viejo. Finalmente, un día el participante le llevó engañado a un centro comercial forzando la situación.

\subsubsection{Dimensión colectiva del cambio de práctica}

Esta dimensión en la segunda etapa está centrada en los cambios que se producen en la responsabilidad en todas sus expresiones. Se dieron cuenta de la importancia que tenían sus decisiones.

En ese escenario en el que todo estaba por construir y se les permitió investigar, trabajaron a través del ensayo-error. Esto fue posible porque les dejó de pesar tanto la opinión pública y se centraron en las personas. Esta metodología les ha permitido descubrir y ajustar su presencia como herramienta puesta al servicio del otro y aprovechar la oportunidad para construir un marco de referencia ajustado a su realidad.

Otro de los cambios que se produjeron fue la distinción entre "mi responsabilidad y la tuya". Que los participantes consuman, pierdan las llaves, pierdan el control de la gestión del piso, etc., deja de alterar a los equipos. Dicho de otro modo, dejaron de actuar en el sentido acción-reacción y tomaron un rol pausado, de observación y escucha, sin juicio. Más bien se prepararon para sostener el dolor de las personas en esos momentos complicados. De hecho, descubrieron que su responsabilidad debía residir principalmente en sus acciones. Por ello, aprovechando la flexibilidad de tiempo y espacio propia del programa (Batle et al., 2020) dedicaron muchas reuniones formales e informales a preparar el acompañamiento. En los casos en los que no pudieron preguntarle directamente a la persona cómo quería ser acompañada, prepararon la acción en base a diferentes hipótesis y siempre pensando en los gustos, necesidades y deseos de la persona. Este ensayo-error y el trabajo a partir de hipótesis convierte a estos equipos en pioneros en nuestro territorio en pos del cambio de modelo en la atención a personas en situación de sin hogar, evidenciándose así la poca atención que se ha prestado a la acción de los profesionales.

Los equipos dejaron de posponer respuestas y flexibilizaron sus prácticas para poder hacer frente al cúmulo de sorpresas o situaciones inéditas propias de la vida cotidiana. De hecho, utilizaron las oportunidades pedagógicas que el mismo entorno les brindó. Un profesional relató un momento en el que un participante fue a cruzar en rojo un semáforo. A partir de ahí, trabajaron la impulsividad y la paciencia.

Asumir la responsabilidad que les corresponde fue más difícil en otras ocasiones, especialmente en la expulsión de un participante de la vivienda. El hecho de la proximidad, la mirada sin juicio y creer en la capacidad de la persona para iniciar un proceso de recuperación hizo que las decisiones se pospusieran varios meses, hasta que finalmente entendieron que hacerlo era poner unos límites sanos para la persona, pues no se la dejaba de acompañar en la vida pero salía del piso a la espera de querer asumir los compromisos propios de la filosofía Housing First. La coordinación con el equipo de salud se fue equilibrando a través de aprendizajes mutuos. Siguiendo con el ejemplo anterior, el equipo de salud entendió que la demanda que tenían de los equipos sociales se debía a una falta de formación previa y especialmente conjunta. Los equipos sociales, por su parte, aprendieron de estos la diferencia entre urgencia y emergencia. Este aprendizaje vino de la mano de la desresponsabilización de las acciones de los participantes. De esa separación entre lo tuyo y lo mío.

Durante esta segunda etapa, se consolida otro de los temas fundamentales para la salud del equipo: los cuidados. Precisamente el trabajo de proximidad, una filosofía innovadora y el hecho de ser un programa piloto hacen que sea más necesario que en otros servicios de atención. Así lo recalcan las experiencias exploradas de Canadá, Marsella y Lisboa. El rol del coordinador ha sido muy importante para garantizar el espacio y los tiempos apropiados. Ambos dejaron de trabajar con objetivos y pasaron a hacerlo con procesos, sin exigir resultados a los equipos sino pequeños detalles que formasen un gran cambio. Tal como señalan en un estudio de la ciudad de Nueva York (Barrenger, Stanhope y Atterbury, 2015), en las reuniones de equipo se generó una identidad grupal a 
través de la relación establecida con los participantes. Los profesionales celebraron cualquier logro de las personas, por pequeños que fueran. Llegaron a alegrarse porque alguien se saludase con un vecino, pidiera en la barra de un bar o les preguntara cómo estaban. Otros elementos que generaron identidad fue el uso del humor como estrategia para destensar situaciones y para cuidarse.

Los espacios de cuidados formales fueron las reuniones de equipo, las de casos, la supervisión y la intervisión, las formaciones y el apoyo de servicios externos para situaciones complicadas. Los informales se dieron después de trabajar o en el descanso, encuentros espontáneos en barrios de la ciudad o viajes en transporte público. Estos espacios sirvieron para que los miembros pudiesen conocerse más, coger confianza y cerciorarse de que no serían juzgados en el contexto laboral. La coordinadora de Montreal apuntaba cómo dejar la puerta de su despacho siempre abierta y estar en él en horas clave servía como estrategia de cuidados informales. Esta plena disposición del estar disponible también aparece en Barcelona.

\subsection{Final del programa: materialización de los cambios}

\subsubsection{Dimensión individual del cambio de práctica}

\section{Personal}

En esta fase, la trayectoria laboral superaba los tres años. Al volver la vista atrás, algunos profesionales reconocieron haber vivenciado momentos que modificaron su vida privada, se identificaban con otras cosas. Al acompañar en la vida cotidiana pudieron experimentar la violencia institucional que recibían las personas. Su sensibilidad al mundo aumentó. Todo esto provocó cambios en su identidad personal. El equipo de Montreal apuntó que algunos profesionales ya no pudieron volver a trabajar con otras metodologías, otros cambiaron de profesión. En el caso de Barcelona, algunos profesionales han relatado cómo esta proximidad les ha hecho replantearse muchas cosas de su vida privada. Incluso el tipo y la calidad de relaciones con los amigos y la familia.

\section{Profesional}

En relación con el estatus profesional, se identifica una aceptación del rol que se había desplegado anteriormente, así como de las consecuencias que pudo tener en las personas la discrecionalidad de ciertas decisiones. Darse cuenta de esto ha provocado que ciertos sectores defiendan el Housing First como modelo único para atender a la población sinhogarista. También que Administraciones americanas y europeas retiren los fondos destinados al modelo anterior. Desde la experiencia canadiense, la coordinadora de Diogène alerta de este peligro. Cada modelo tiene funciones y destinatarios diferentes. Ahora bien, lo que sí ha salido a la luz es la necesidad de revisión de las formas de hacer del modelo en escalera. En Barcelona ambas gestoras sociales han aplicado cambios metodológicos en otros programas de atención, flexibilizando horarios y adaptando los servicios residenciales a las personas. Los beneficios de la práctica reflexionada han sido evidentes en el rol profesional.

Los profesionales trabajaron desde la coherencia y la permeabilidad entre lo personal y lo laboral, poniendo en evidencia la parte de defensa de los derechos de las personas (advocacy) como ciudadanas. Además, han visto cómo esta práctica fue reparadora para las personas, que venían con un cúmulo brutal de desconfianza al profesional. Siguiendo a Christopher McAll (2008), esta manera de trabajar puede romper con las interacciones, que lejos de reconocer los efectos de las discriminaciones y estigmas, reproducen desigualdades.

En definitiva, el rol ostentado por los profesionales es completamente novedoso. Desde la otra investigación cualitativa (Llobet et al., 2020) centrada en los efectos del programa sobre la recuperación de las personas, se puede constatar el impacto emocional que también ha tenido la relación para los participantes. Según los relatos, ha sido una experiencia que los ha transformado como profesionales.

\subsubsection{Dimensión colectiva del cambio de práctica}

Los equipos se han consolidado como una unidad muy fuerte con identidad propia, al servicio del proceso de recuperación de las personas y al mismo tiempo al servicio de sus propios compañeros de equipo. Claramente con complicidad, y eso se notó en la autonomía que demostraron a la hora de tomar decisiones. Lo hicieron unilateralmente cuando tenían la certeza de la posición del equipo frente a ese tema. Mostraron disponibilidad para apoyarse, escucharse e intercambiarse en visitas si alguien se veía sobrepasado.

Uno de los cambios más importantes en esta etapa fue la consolidación de ciertas pautas para la práctica asociadas a situaciones. Por ejemplo, tras un tiempo sin poder contactar con una persona, solo podía entrar en el piso con la policía y esta siempre primero. Al enseñar los pisos a los participantes se les dieron las llaves para que fuesen ellos quienes abrieran la puerta. El primer caso se estableció a partir del deceso de un participante en su vivienda y el consecuente shock de los profesionales que entraron. La segunda decisión fue fruto de hacerlo una vez y ver el efecto que tuvo en la persona abrir la puerta de su futura casa. Son pautas de acción inductivas y flexibles adaptables a cada persona y situación.

La práctica reflexionada ha permitido al equipo proveerse de espacios para conversar, compartir, cuidarse y dudar; en definitiva, transitar. $\mathrm{Ha}$ 
permitido que se generase una identidad colectiva y unos niveles de confianza muy altos. Esta confianza es la que necesita el equipo para poner a la persona en el centro, de la misma manera que los participantes necesitan tener confianza en los profesionales para iniciar el proceso de recuperación acompañados. Se evidencia aquí la dimensión intersubjetiva de la práctica.

\section{Otras influencias sobre la práctica}

\subsection{La influencia de la investigación sobre el cambio de práctica}

Esta investigación cualitativa no se ha diseñado como un dispositivo de acompañamiento al proceso de transformación dentro del programa Primer la Llar, sino que se concibieron de forma separada. A pesar de no tener una presencia constante, ni estar pensada para promover el cambio sino para documentarlo, ha tenido influencia directa sobre la transformación de la práctica.

Al principio, y debido a la novedad de este tipo de investigaciones, los equipos dijeron no entender la utilidad de la presencia de las investigadoras. Sin embargo, con el tiempo expresaron que estos espacios reflexivos les dieron la posibilidad de dar sentido a lo que hacían.

Las entrevistas grupales han formalizado un espacio de verbalización. Un ejercicio que ha estructurado el conocimiento y lo ha hecho transmisible. Recogerlo desde el relato permite captar el ir y venir de la acción-reflexión, el ensayo-error y los ajustes propios de la innovación. Este proceso ha pasado por diversas etapas, algunas más amables, otras más amargas. Para que esta verbalización fluyera, se ha tenido que trabajar la relación de confianza generada entre los investigadores y el equipo. Al sentir que no había juicio, compartieron pensamientos y sentimientos muy íntimos.

Siguiendo a Tilman (1996), es de ilusos pensar que una nueva metodología aparece siendo operativa y lista para aplicarse. Documentar el proceso es un acto de responsabilidad y reconocimiento a los profesionales y su savoir en usage o saberes situados. Durante las entrevistas, los profesionales han expresado sentirse valorados como fuentes propias de conocimiento.

\subsection{La gobernanza}

Más allá de las lógicas implicaciones en la gestión y desarrollo operativo que toda gobernanza debe cubrir en el desarrollo de cualquier empresa, esta cumple una función básica, primigenia y determinante: la gobernanza es la primera materialización en pos de la consecución del objetivo final -es decir, del cumplimiento de la misión-.
La gobernanza construye la estructura sobre la que se sustentará, primero en futuro, después en vivo, el cómo -es decir, de qué manera, con que métodos-; el con qué recursos -humanos, tecnológicos, materiales-; el con quién -qué alianzas deseables, qué alianzas imprescindibles, qué perfil de profesionales e instituciones- se va a llevar a cabo la misión.

Si eso es central en todo proyecto, lo es con más intensidad cuando este no dispone de precedentes y tiene la auténtica consideración de experimentar, como ha sido el caso.

En este sentido, la gobernanza de Primer la Llar ha sido también un work in progress que se fundamentó en el esfuerzo del Ayuntamiento de Barcelona por contar con todos los elementos a su alcance a partir del escaso conocimiento previo disponible sobre el modelo, como lo demuestra el pliego de condiciones técnicas inicial que, en 2015, se comprometía con detalle en que había de ser un proyecto basado en el modelo Housing First.

En cualquier caso, la condición absolutamente experimental del modelo y la ausencia de conocimiento previo sobre sus desarrollos en ubicaciones en contexto europeo o extracomunitario, no permitió el diseño de una gobernanza que considerase aspectos críticos como las alianzas imprescindibles a nivel de otros sistemas ajenos al social, como el de salud y el de vivienda, a nivel de subniveles del sistema propio de servicios sociales, entre servicios de primaria y secundaria. Tampoco las alianzas necesarias, como hubiesen sido las relacionadas con el mercado libre de vivienda de Barcelona, no fueron consideradas. Tampoco pudieron ser considerados los tiempos a partir de los cuales planificar la consecución de los objetivos de gobernanza en los momentos adecuados para un correcto acompañamiento en el desarrollo del proyecto - por ejemplo, algunos de los objetivos de gobernanza que deberían haber sido alcanzados con anterioridad a determinados momentos de la operativa del proyecto generaron importantes alteraciones en el desarrollo del modelo-.

El otro gran ámbito sobre el que la falta de conocimiento previo afectó en el diseño de una mejor gobernanza fue el modelo en sí mismo y sus implicaciones: lo que estos años de aplicación del modelo ha demostrado en relación con el rotundo cambio de modelo que implica el modelo Housing First a participantes, profesionales, sistema de servicios sociales y sistema de salud, fue descubierto sobre la marcha y adaptado conforme la práctica generaba conocimiento para ser aplicado.

A su vez, ha tenido afectación en una falta de posición sobre cuestiones importantes surgidas a partir del desarrollo del proyecto: el perfil profesional del par igual (peer worker); la posición como proyecto frente al tratamiento asertivo comunitario y la gestión intensiva de casos (ACT e ICM, respectivamente, por sus siglas en inglés) en relación 
con el trabajo entre los equipos sociales y de salud mental; la comprensión del abanico de modelos de acompañamiento basados en los formatos Housing Led como áreas de especialidad en atención a perfiles o necesidades específicas; y la aún no plena conciencia sobre las implicaciones prácticas de Primer la Llar en relación con el modelo de atención centrada en la persona, elementos todos ellos que a partir de los descubrimientos.

En cualquier caso, todos estos elementos han sido identificados y se ha ido avanzando de manera consciente en la mayoría de ellos, generando así un avance conceptual que seguramente los equipos responsables de la gobernanza en el Ayuntamiento de Barcelona deberán transmitir y hacer comprender a sus grupos de decisión y de interés para poner a su entorno al nivel de desarrollo de conocimiento de las posibilidades del modelo Housing First del que ahora dispone.

\section{Conclusiones}

Esta investigación pone en evidencia el interés por investigar la práctica profesional en general, y específicamente sobre el ámbito del sinhogarismo, a través del análisis de la implementación del innovador modelo Housing First.

La pertinencia de poner en marcha un dispositivo de análisis de la práctica para observar, analizar y documentar su transformación; la utilidad del relato de práctica como método para compartir las acciones pasadas; cómo el mismo proceso de relatarlas deviene un acto reflexivo sobre la práctica y que cuando las investigadoras comparten en un segundo momento un análisis-devolución de las narrativas, abre una reflexión de segundo orden en el equipo.

A nuestro entender, son varios los elementos que activan el proceso de transformación de la práctica en relación con lo que venían haciendo con anterioridad los profesionales. El carácter piloto del programa Primer la Llar y el mandato explícito para poder experimentar, a pesar de imprimir una sobreexposición y presión inicial en los equipos, les da el permiso para llevar a cabo un proceso de ensayo-error.

La falta de un marco de referencia sobre la práctica a emprender, así como la falta de una experiencia concreta a la cual consultar, activa en ambos equipos, con procesos diferentes, espacios formales e informales en los que comparten sus dudas e inseguridades, sus aciertos y errores, sus estrategias y habilidades. La creación de estos espacios contribuye a dos movimientos que se realizan en paralelo: a deconstruir los lenguajes y aquello dado por sentado de la práctica anterior, que no se atrevían a interpelar; y a co-construir la nueva práctica, desde una toma de conciencia de sus acciones, lenguajes y cuerpos. Estos dos movimientos cohesionan a los equipos, desde el saber-hacer del coordinador/a.
Los resultados ponen de relieve cómo la práctica no solo está comprendida por saberes académicos y técnicos, sino también por las experiencias de vida de los propios profesionales. Cómo sujetos que también han sido atravesados por situaciones biográficas diversas y cómo estas vivencias han facilitado la comprensión de los principios del Housing First y la activación y creación de una relación intersubjetiva, de proximidad y confianza con los y las participantes de este programa.

Del análisis de los tres momentos se desprende que el trabajo y el individuo en tanto que profesional no son elementos estancos. La práctica reflexiva a nivel grupal los lleva a pensar su acción y a ser autocríticos a nivel individual y esta confluencia explica el cambio de rol e identidad de los profesionales. El poder de los profesionales se va transformando de forma consciente desde el reconocimiento mutuo hacia una mayor horizontalidad de la relación. También constatamos la importancia del afecto como metodología que repara los efectos de las discriminaciones y el estigma y reduce la distancia social, generando nuevos dilemas a los profesionales ante la hospitalidad o aceptando ser invitados por los y las participantes.

La transformación de la práctica requiere de un compromiso personal con el otro, un re-pensarse en la relación intersubjetiva y transformarse desde las múltiples posibilidades que ofrecen los espacios de encuentro desde la vida cotidiana de la persona a través de tiempos largos, más de tres años que ofrece este programa. La práctica dialógica permite pasar de la jerarquía del conocimiento experto inicial a reconocer las fortalezas y conocimientos de las personas.

El acompañamiento desde la vida cotidiana deja de ser funcional para pasar a ser existencial, e imprime lógica de proceso y coherencia a la práctica. También coloca a los profesionales ante situaciones singulares e inesperadas, que responden de forma pensada, evitando precipitarse y reduciendo así el error o daño que podrían causar. Cuando se dan cuenta de que se han equivocado, rectifican y lo conversan con la persona. La atención centrada en la persona ha sido un resultado que ha emergido de forma inductiva desde la personalización de la atención a lo largo del programa, a través de las adaptaciones y cambios que han ido realizando.

Sin embargo, la proximidad y la exposición emocional que requiere este tipo de acompañamiento, que debe sostener el dolor y sufrimiento en momentos de crisis, puede llegar a desbordar a los profesionales, mostrando la importancia de la formación previa y continuada que requiere este tipo de programa, así como los espacios de cuidado que deben activarse por parte del coordinador/a para proteger al equipo. El uso del humor también es una estrategia para destensar y cuidarse.

El análisis del quehacer profesional en la implementación de este programa piloto muestra 
como la transformación de la práctica es un proceso de indagación artesanal y de reflexión de la acción. Requiere aceptar la incertidumbre, reconocer la incomodidad, humanizar la relación para reconocer a la persona como mecanismo generador de confianza y activar la creatividad necesaria para acompañar el proceso de mejora y recuperación. 


\section{Referencias bibliográficas}

ALFÖLDI, F. (2017) : L'analyse des pratiques en travail social, Malakoff, Dunod.

ANDERSON-BARON, J.T. y COLLINS, D. (2018): “'Take whatever you can get': practicing Housing First in Alberta", Housing Studies, vol. 34, n 1, pp. 1.286-1.306.

ANDVIG, E.S. ; SALLØR, K.T. y OGUNDIPE, E. (2018): «Harm reduction in a Norwegian housing first project: a qualitative study of the treatment providers' practice", Advances in Dual Diagnosis, vol. 11, 1.

ÁVILA, D.; CASSIÁN, N.; GARCÍA, S. y PÉREZ, M. (2019): Por una acción social crítica. Tensiones en la intervención social, Barcelona, Editorial UOC.

BACZKOWSKA, M. (2017): Conclusion: Les moments de l'intervention sociale et leurs acteurs. En: S. GARNEAU y D. NAMIAN (eds.), Erving Goffman et le travail social. Otawa: Les Presses de l'Université d'Ottawa, pp. 280-300.

BARBIER, J.M. (1993): La evaluación en los procesos de formación, Barcelona, Ministerio de Educación y Ciencia.

- (1999): Prácticas de formación. Evaluación y análisis, Buenos Aires, Ediciones Novedades Educativas.

- (2004): "El análisis de las prácticas y las herramientas", Prácticas de formación, evaluación y análisis, Barcelona, Paidós Ibérica, pp. 71-99.

- (2011): Vocabulaire d'analyse des activités, París, Puf.

BARDÓN, J.L. (1998): «Bersoak, la voz que perdura. La actualidad de la literatura oral”, Kikiriki. Cooperación educativa, vol. 47, pp. 78-81.

BARRENGER, S.L.; STANHOPE, V. y ATTERBURY, K. (2015): "Discursive Processes Creating Team Culture and Recovery Orientation Among Housing First
Providers", American Journal of Psychiatric Rehabilitation, vol. 18, n 3, pp. 247-264.

- (2017): "Challenging Dominant Discourses: Peer Work as Social Justice Work", Journal of Progressive Human Services, pp. 1-21.

BATLE, S.; CARTOIXÀ, J.; LLOBET, M. y URIBE, J. (2020): "Ethical Relation and Control: Exploring Limits in Domestic Sphere of Home", European Journal of Homelessness, vol. 13, n 2, pp. 51-72.

BERTAUX, D. (1976): Histoires de vies ou récits de pratiques? méthodologie de l'approche biographique en sociologie, París, Centre d'étude des mouvements sociaux.

- (2005): Los relatos de vida. Perspectiva etnosociológica, Barcelona, Ediciones Bellaterra.

BLUMER, H. (1982): El interaccionismo simbólico: perspectiva y método, Barcelona, Hora.

BOURDIEU, P. (2007): El sentido práctico, Madrid, Siglo Veintiuno.

BUXANT, C. (2018): "The Challenge of Implementing the Housing First Model: How Belgium Tries to Connect Fidelity and Reality", European Journal of Homelessness, vol. 12, n 3, pp. 229-252.

CABRERA, P. (1998): Huéspedes del aire. Sociología de las personas sin hogar en Madrid, Madrid, Universidad Pontificia Comillas.

- (2000): Mujeres sin hogar en España. Informe Nacional para Feantsa, Madrid.

COSENTINO, R.; DEMITA, S.; LENZI, M.; GABOARDI, M.; VIENO, A. y SANTINELLO, M. (2020): "Lo stress lavorativo negli operatori dei servizi dedicati alle persone senza dimora : confronto tra operatori di servizi tradizionali e di servizi di Housing First", Medicina del lavoro, vol. 42, n 3, pp. 174-177. 
DANEL, P.M. (2020): "Habitar la incomodidad desde las intervenciones del trabajo social”, Revista de trabajo social y ciencias sociales, vol. 31, pp. 1-13.

DE ROBERTIS, C. (2013): “Pratique professionnelle : une tentative de définition», Institutions, acteurs et pratiques dans l'histoire du travail social, Rennes, Presses de l’EHESP, pp. 137-144.

DELORY-MOMBERGER, C. (2014): “Experiencia y formación. Biografización, biograficidad y heterobiografia", Revista Mexicana de investigación educativa, vol. 19, no. 62, pp. 695-710.

DESGAGNÉ, S. (2005): Récits exemplaires de pratique enseignante. Analyse typologique, Québec, Presses de l'Université du Québec, disponible en: 〈www.puq.ca〉.

DEVINE, G. y BERGIN, M. (2020): “Experiences of Frontline Workers' Engagement with Mental Health Services for Homeless Adults in Ireland", European Journal of Homelessness, vol. 14, no. 2, pp. 129-147

EISNER, E.W. (1998): El ojo ilustrado: indagación cualitativa y mejora de la práctica educativa, Barcelona, Paidós Ibérica.

ESTEBAN, M.L. (2004): Antropología del cuerpo: género, itinerarios corporales, identidad y cambio, Barcelona, Bellaterra.

FABLET, D. (2004): «Les groupes d'analyse des pratiques professionnelles: une visée avant tout formative», Connexions, vol. 82, pp. 105-117.

- (2007) : «Formation des travailleurs sociaux et analyse des pratiques professionnelles", Revue internationale des sciences de l'education, vol. 17, pp. 39-50.

FENWICK, K.; HENWOOD, B.; LENGNICK-HALL, R.; STEFANCIC, A. y GILMER, T. (2019): «Exploring Variation in Housing First Implementation: The Role of Fit", Human Service Organizations Management, Leadership and Governance, vol. 43, no. 5, pp. 392-406.

FREIRE, P. (2005): Pedagogía del oprimido, Buenos Aires, Siglo veintiuno editores.

GARCÍA-MOLINA, J. y SAÉZ, J. (2011): “Educación Social ¿Qué formación para qué profesional?”, Revista de Educación Social, vol. 13, pp. 1-14.

GARNEAU, S. y NAMIAN, D. (2017): Erving Goffman et le travail social, Montreal, Les Presses de l'Université d'Ottawa.

GHERARDI, S. (2008): "Situated Knowledge and Situated Action”, en: D. BARRY y H. HANSEN (eds.): The SAGE Handbook of New Approaches in Management and Organization, Londres, SAGE Publications, pp. 516-525.

GOFFMAN, E. (1991): Los momentos y sus hombres, Barcelona, Paidós comunicación.

GRIMAUD, L. (2003): «Le récit de pratique, un outil clinique en institution?», VST - Vie sociale et traitements, vol. 3, pp. 8-11.

GUIGUE, M. (2002): «Analyse de pratiques», Recherche \& Formation, vol. 39, n. 1, pp. 107-121.

HAIDET, P. (2007): “Jazz and the 'art' of medicine: Improvisation in the medical encounter",
Annals of Family Medicine, vol. 5, n. 2, pp. 164-169.

HASFORD, J.; NELSON, G.; WORTON, S.K.; MACNAUGHTON, E.; MACLEOD, T.; PIAT, M.; TSEMBERIS, S.; STERGIOPOULOS, V.; DISTASIO, J.; AUBRY, T. y GOERING, P. (2019): “Knowledge translation and implementation of housing first in Canada: A qualitative assessment of capacity building needs for an evidence-based program", Evaluation and Program Planning, vol. 75, pp. 1-9.

HOMELESSLINK (2017): Guidance for Support providers, London.

JAMOULLE, P.(2007): «La proximité», Les politiques sociales, vol. $3 \& 4$, pp. 42-59.

LABORDE, D. (1999): «Enquête sur l'improvisation», en: M. de FORNEL y L. QUÉRÉ (eds.): La logique des situations, París, Éditions de l'École des Hautes Études en Sciences Sociales, pp. 261-299.

LAFORTUNE, L. (2009): Professional competencies for accompanying change. A frame or reference, Québec, Presses de l'Université du Québec.

LENZI, M.; SANTINELLO, M.; GABOARDI, M.; DISPERATI, F.; VIENO, A.; CALCAGNI, A.; GREENWOOD, R.M.; ROGOWSKA, A.M.; WOLF, J.R.; LOUBIÈRE, S.; BEIJER, U.; BERNAD, R.; VARGAS-MONIZ, M.J.; ORNELAS, J. ; SPINNEWIJN, F. y SHINN, M. (2020): «Factors Associated with Providers’ Work Engagement and Burnout in Homeless Services: A Cross-national Study", American Journal of Community Psychology, vol. 0, n. 1, pp. 1-17.

LLOBET, M. y AGUILAR, M. (2016): “El Housing First. El dret a l'habitatge dels més vulnerables", Barcelona Societat, vol. 20, pp. 16-32.

LLOBET, M.; BOIXADÓS, A.; CARTOIXÀ, J.; MUÑOZ, A.; SANCHO, J.; SERRANO, M. (2020): Informe final del Programa Primer la Llar. Als 40 mesos d'estada a l'habitatge, Ayuntamiento de Barcelona, Barcelona.

LO SARDO, S. (2016): Sorties de rue. Une ethnographie des pratiques d'intervention Housing First, Bruselas.

MARCEL, J.F.; OLRY, P.; ROTHIER-BAUTZER, E. y SONNTAG, M. (2002): «Note de synthèse : Les pratiques comme objet d'analyse», Revue française de pédagogie, vol. 138, pp. 135-170.

MCALL, C. (2008): «Trajectoires de vie, rapports sociaux et production de la pauvreté», en V. CHATÊL y S. ROY (eds.): Penser la vulnérabilité. Visages de la fragilisation du social, Québec, Presses de l'Université du Québec.

MEIRIEU, P. (1998): Frankenstein educador, Barcelona, Editorial Laertes.

NAVARRO, S. (2015): “En busca del fuego en la intervención social: vindicación de lo comunitario, artesanía de lo relacional”, El Servicio Social Municipal del Ayuntamiento de Bilbao, hacia un modelo más comunitario y relacional, Bilbao, Rayuela creativa, pp. 12

NAVARRO, S. (2017): Saber femenino, vida y acción social. Madrid, Editorial CCS.

PADGETT, D.K. (2012): “Choices, Consequences and Context : Housing First and its Critics", 
European Journal of Homelessness, vol. 7, no. 2, pp. 341-347.

PERRENOUD, P. (2012): “Le travail sur l'habitus dans la formation des enseignants", Former des enseignants professionnels: Quelles stratégies? Quelles compétences?, De Boeck Supérieur, pp. 211-237.

PESSIN, A. (2004) : Un sociologue en liberté. Lecture de Howard S. Becker, Québec, Presses de l'Université Laval.

PIAGET, J. (2000): Biología y conocimiento. Ensayo sobre las relaciones entre las regulaciones orgánicas y los procesos cognoscitivos, México D.F., Siglo XXI.

RICOEUR, P. (1990): Soi-même comme un autre, París, Seuil.

- (2004): Tiempo y narración, Madrid, Siglo XXI.

ROGERS, J.; GEORGE, T. y ROBERTS, A. (2020): “Working with individuals who have experienced homelessness: Stresses and successes", Housing, Care and Support, vol. 23, no. 2, pp. 65-76.

ROSA, F. (2019): Less? No more. Buone pratiche per uan progettazione di interventi di Supportive Housing destinati all'accoglienza delle persone senza dimora in Italia, Politécnico de Torino.

SACCO, V. (2020): A missing piece of the puzzle: Health care providers' experiences supporting older homeless adults in outreach settings, University of Waterloo.

SAHLIN, I. (2005): "The Staircase of Transition Innovation”, The European Journal of Social Science Research, vol. 18, n. 2, pp. 115-136.

SAMHSA (2010): Permanent Supportive Housing: Training Frontline Staff, Rockville, Center for Mental Health Services, Substance Abuse and Mental Health Services Administration, U.S. Department of Health and Human Services.

SAN MARTÍN, D. y QUILAQUEO, D. (2012): “Habitus profesional y relaciones intersubjetivas entre profesores principiantes y experimentados", Perfiles Educativos, vol. 34, n. 136, pp. 63-78.

SANTIBÁÑEZ, C. (2012): “Teoría de la argumentación como epistemología aplicada”, Cinta de Moebio, vol. 43, pp. 24-39.

SCHÖN, D.A. (1998): El profesional reflexivo: cómo piensan los profesionales cuando actúan, Barcelona, Paidós.

SCHÖN, D.A. y ARGYRIS, C. (1995): Organizational learning II: Theory, method and practice, Addison Wesley.

SCHÖN, D.A. y REIN, M. (1994): Frame Reflection: Toward the Resolution of Intractable Policy Controversies, Nueva York, Basic Books.

SENNETT, R. (2009): El artesano, Barcelona, Editorial Anagrama.

STANHOPE, V. (2012): “The ties that bind: Using ethnographic methods to understand service engagement”, Qualitative Social Work, vol. 11, n. 4 , pp. 412-430.

TILMAN, F. (1996): «Comment parler de sa pratique pour la faire partager?», Courrier du CETHES, vol. 35 , pp. 18-28.

VÁNĚ, J. (2020): “Merit versus Necessity: Housing First and its Forms in Practice", European Journal of Homelessness, vol. 14, n 1, pp. 55-78.

VERGNAUD, G. (1996): «Au fond de l'action, la conceptualisation", Savoirs théoriques et savoirs d'action, París, Presses Universitaires de France.

WATSON, D.P.; AHONEN, E.Q.; SHUMAN, V.; BROWN, M.; TSEMBERIS, S. y HUYNH, P. (2018): «The housing first technical assistance and training (HFTAT) implementation strategy: outcomes from a mixed methods study of three programs", Substance Abuse Treatment, Prevention, and Policy, vol. 13, n 32, pp. 1-13. 